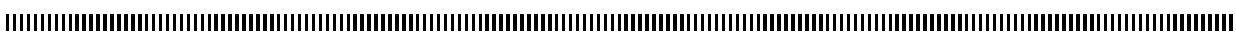

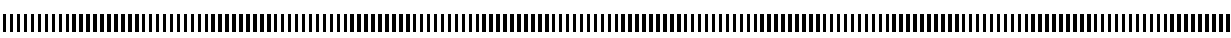
|

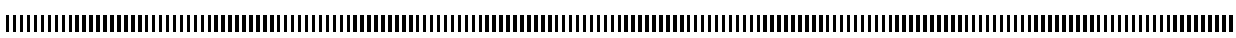

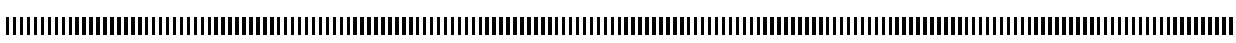
| |

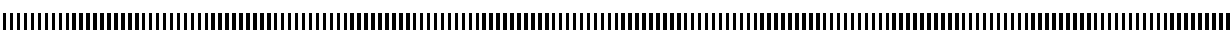

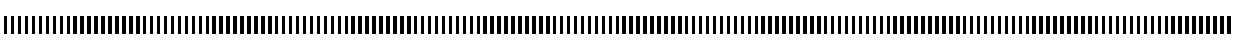
|

2007 International Conference in Honor of Claude Lobry

\title{
La méthode des élucidations successives
}

\author{
Claude Lobry \\ INRIA-Sophia Antipolis et \\ Université de Nice Sophia-Antipolis \\ Nice \\ France \\ claude.lobry@inria.fr
}

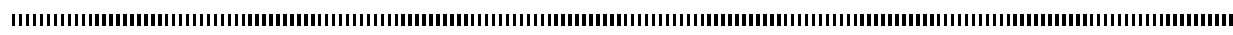

RÉSUMÉ. Dans le processus d'élaboration d'un modèle on insiste beaucoup sur la nécessité de confronter le modèle à la réalité qu'il est sensé représenter. II est un autre aspect de la modélisation, à mon avis tout aussi essentiel, dont on ne parle pas. II s'agit d'un travail logico-linguistique où des modèles formels sont utilisés pour produire des prédiction qui ne sont pas confrontées à la réalité mais servent à falsifier des affirmations qui semblaient pourtant se déduire du modèle. Plus précisément un premier modèle informel est décrit dans la langue naturelle et, toujours dans la langue naturelle, semble dire quelques chose mais de façon plus ou moins claire. Alors on traduit le modèle informel en un modèle formel (mathématique ou informatique) où ce qui était argumentation devient démonstration. Le modèle formel sert ainsi à lever des ambiguïtés de la langue naturelle. Mais inversement un texte trop formalisé perd rapidement tout sens pour un cerveau humain ce qui rend nécessaire le retour à une langue moins formelle. Ce sont ces "traductions" successives entre langues plus ou moins formelles que je cherche à analyser sur deux exemples, le premier en dynamique des populations, le second en mathématiques.

ABSTRACT. In the process of elaboration of a model one emphasize on the necessity of confronting the model with the reality which it is supposed to represent. There is another aspect of the modelling process, to my opinion also essential, about which one usually do not speak. It consists in a logico-linguistic work where formal models are used to produce prediction which are not confronted with the reality but serve for falsifying assertions which nevertheless seemed to be derived from the not formalized model. More exactly a first informal model is described in the natural language and, considered in the natural language, seems to say some thing but in a more or less clear way. Then we translate the informal model into a formal model (mathematical model or computer model) where what was argumentation becomes demonstration. The formal model so serves for raising ambiguities of the natural language. But conversely a too much formalized text quickly loses any sense for a human brain what makes necessary the return for a less formal language. It is these successive "translations" between more or less formal languages that I try to analyze on two examples, the first one in population dynamics, the second in mathematics.

MOTS-CLÉS : Analse non standard, dynamique des populations, modèle, théorie des bifurcations.

KEYWORDS : Bifucation theory, model, non standard analysis, population dynamics.

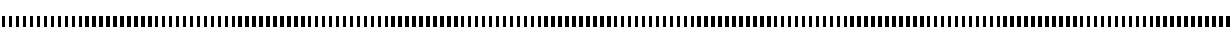




\section{Introduction}

La "modélisation" est à la mode, sans qu'on sache vraiment ce que ce mot recouvre. Quand on en parle il est de bon ton de prononcer des phrases du genre :

- "Le modèle n'est pas la réalité",

- "Il faut identifier les paramètres du modèle",

- "Un modèle doit être validé",

qui sont là pour affirmer que leur auteur souscrit aux grands principes de la méthode hypothético-déductive et donc qu'il est un scientifique respectable puisqu'il ne confond pas ses fantasmes avec le monde réel. On ne peut que souscrire à de telles banalités mais je pense que ce n'est pas tout. Dans la modélisation je vois aussi - et peut-être principalement - fonctionner le processus suivant :

1) ...un texte numéro $n$ est écrit, interprété et commenté.

2) Les commentaires soulèvent des questions, des doutes sur l'interprétation du texte numéro $n$ voire sur le texte lui même.

3) Les commentaires et les doutes sont formalisés et l'on fait fonctionner la mécanique formelle pour en déduire.....

4) ....un texte $n+1$ et ses commentaires qui clarifient le texte $n$ et ses commentaires mais soulèvent à leur tour des questions....

où, on le constate, le retour, à travers l'expérience, n'est pas présent. Ce n'est donc qu'une activité de clarification du langage, un processus d'élucidation. Ce processus qui n'a pas de début ni de fin, je l'appelle processus d'élucidations successives et son emploi conscient méthode des élucidations successives ${ }^{1}$. C'est à une clarification des point 1 à 4 , notamment des mots soulignés et des petits points, que je voudrais me livrer ici. A cette fin je commence par donner deux exemples tirés de ma pratique professionnelle où je vois ce processus à l'oeuvre et ensuite je commente à la lumière de ces exemples.

\section{Deux exemples}

Pour les deux exemples je suis le même plan : une description du contexte, le problème, les mathématiques associées au problème, leur résolution et, enfin, un résumé de l'exemple en quelques lignes. Chaque exemple est suivi de notes qui contiennent des renvois bibliographiques.

\subsection{Exemple 1 : Le paradoxe de l’inversion de la compétition}

\subsubsection{Le contexte}

En écologie (scientifique, faut-il le préciser?), comme en physique, il existe des "principes" et des "lois". Par exemple il existe une loi empirique qui relie la richesse spécifique d'un habitat à sa superficie, comme par exemple la relation de la figure (2.1.1), qui est expliquée par la théorie de l'équilibre dynamique de MacArhur et Wilson [2]. Tout ceci

1. Je dis bien "élucidations", pas "élucubrations successives" :=). 


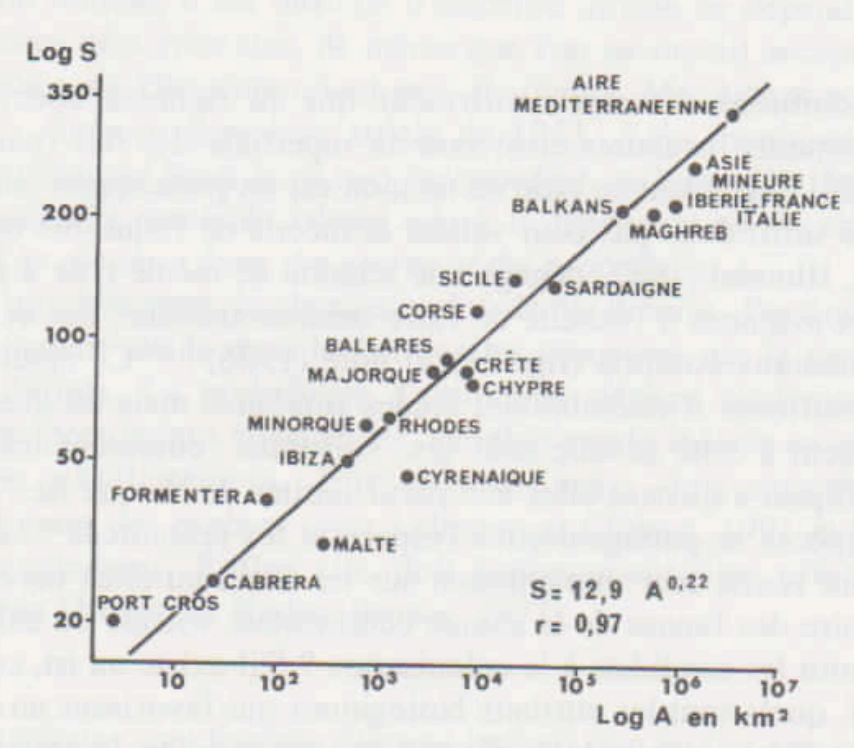

Figure 1. Relation log-log entre le nombre d'espèces d'oiseaux nicheurs et la superficie des territoires insulaires et continentaux dans l'aire méditerrannéenne. (d'après Barbault [1] p. 50

est assez semblable à ce que l'on peut rencontrer en physique.

En revanche il existe des principes, comme celui de l'exclusion compétitive,

Deux espèce qui exploitent une même ressource dans un environnement stable ne peuvent coexister à l'équilibre, la plus apte éliminant l'autre

qui n'ont pas le même statut. En effet, on se doute, qu'énoncé aussi généralement que ci-dessus, un tel principe trouve facilement des contre-exemples. De fait, ce principe a alimenté de longues discussions qui ne sont pas terminées ${ }^{2}$. L'exemple que je vais développer se situe dans ce contexte de la compétition entre deux espèces.

\subsubsection{Qui pourrait être une loi informelle de l'écologie}

On s'intéresse à une situation où deux espèces, $\mathrm{A}$ et $\mathrm{B}$, sont en compétition. Cette compétition s'exerce dans un espace qui possède diverses particularités qu'on appellera "environnement" et qui sera noté $\mathrm{E}$. On dit que l'espèce $\mathrm{B}$ élimine l'espèce $\mathrm{A}$ si, au cours du temps, la taille de la population des individus de l'espèce A diminue jusqu'à ce que l'élimination intervienne. On dira, dans ce cas, que "l'environnement E est favorable" à l'espèce B. Un environnement est rarement fixe; il évolue au cours du temps. Imaginons que nos deux espèces subissent périodiquement deux environnements différents E1 et E2 et que ces deux environnements soient tous les deux favorables à B. Nous concluons que la succession E1, E2, E1, E2, E1, etc... des deux environnements est elle aussi favorable à $\mathrm{B}$ ce que nous pouvons énoncer sous la forme d'une proposition :

2. II y a près de cinquante ans, Hardin publiait un long article [3] dans Sciences: The Competitive Exclusion Principle, sur les origines de l'expression et du concept. 
Proposition 2.1 Si E1 et E2 sont des environnements favorables à l'espèce B alors une succession régulière d'environnements E1,E2, E1, E2,... est aussi favorable à l'espèce $B$.

Est-ce bien certain? A priori on ne voit pas très bien comment des successions "favorables" à l'espèce B pourraient ne pas lui être "favorable". Toutefois un doute peut naître en raison de l'usage du mot "favorable" qui désigne un processus temporel au cours duquel B finit par éliminer A et que la succession des environnements favorables est elle aussi est un processus. Il y a succession de processus, en quelque sorte un "processus" de processus. La logique élémentaire s'applique-t-elle dans ce cas? Il y a doute. Une façon d'aborder ce doute est de construire un "modèle".

\subsubsection{Un modèle mathématique}

Ces questions de compétition entre espèces ont été étudiées depuis longtemps, entre autres par Volterra qui a proposé les premiers modèles. Je commence par rappeler ce que dit le modèle de compétition de Volterra. Soit le système de deux équations :

$$
\left\{\begin{array}{l}
\frac{d x}{d t}=\alpha(1-a x-b y) x \\
\frac{d y}{d t}=\beta(1-c x-d y) y
\end{array}\right.
$$

où $x$ et $y$ représentent respectivement la taille des populations d'individus d'espèce $\mathrm{A}$ et $\mathrm{B}$ et où les constantes sont toutes positives. Une des prédictions de ce modèle est que si :

$$
\begin{aligned}
& a>c \\
& b>d
\end{aligned}
$$

l'espèce $\mathrm{B}$ gagne la compétition. La démonstration mathématique en est très aisée, tout juste du niveau d'une licence de sciences de la vie. Les constantes $a$ et $b$ expriment la "force" de la compétition exercée par l'espèce $\mathrm{A}$ sur elle même et exercée par l'espèce $\mathrm{B}$ sur l'espèce $\mathrm{A} ; c$ et $d$ ont une interprétation symétrique. La prédiction de ce modèle ne nous surprend donc pas : Si les pressions exercées sur A sont les plus fortes, A perd la compétition.

Nous considérons les deux instantiations du modèle (1) ci-dessus, sensées représenter deux environnements :

\section{Environnement I}

$$
\left\{\begin{array}{l}
\frac{d x}{d t}=1(1-2 x-2 y) x \\
\frac{d y}{d t}=\frac{5}{2}\left(1-\frac{3}{2} x-\frac{3}{2} y\right) y
\end{array}\right.
$$

\section{Environnement II}

$$
\left\{\begin{array}{l}
\frac{d x}{d t}=\frac{5}{2}\left(1-\frac{3}{4} x-\frac{3}{4} y\right) x \\
\frac{d y}{d t}=1\left(1-\frac{1}{2} x-\frac{1}{2} y\right) y
\end{array}\right.
$$

Ces environnements sont donc tous les deux favorables à l'espèce B. 


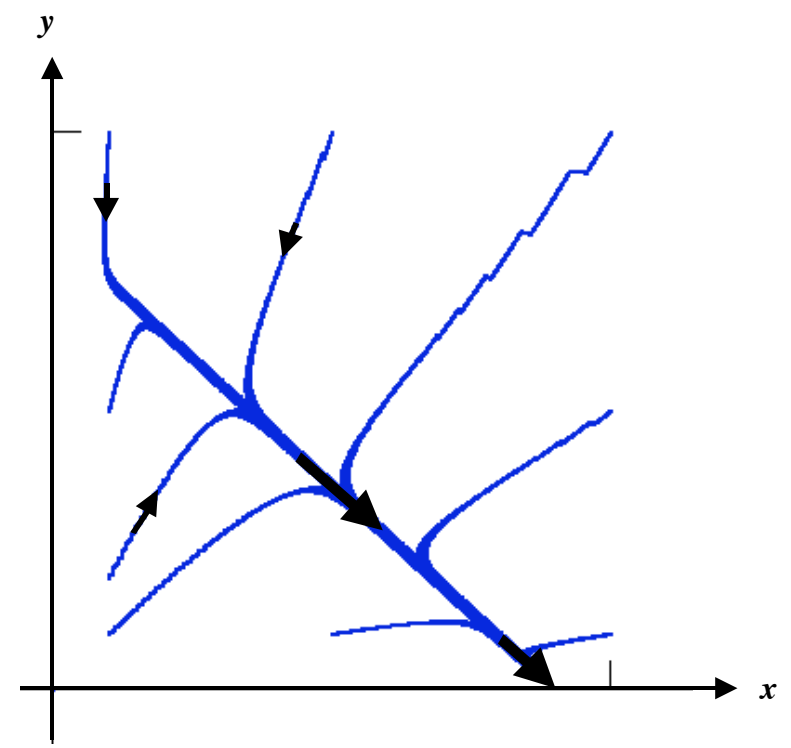

Figure 2. Compétition en environnement périodique

\subsubsection{Une simulation surprenante}

Sur la figure (2) nous voyons le résultat d'une simulation du système :

$$
\left\{\begin{array}{l}
\frac{d x}{d t}=u(t) 1(1-2 x-2 y) x+\left(1-u(t) \frac{5}{2}\left(1-\frac{3}{4} x-\frac{3}{4} y\right) x\right. \\
\frac{d y}{d t}=u(t) \frac{5}{2}\left(1-\frac{3}{2} x-\frac{3}{2} y\right) y+(1-u(t)) 1\left(1-\frac{1}{2} x-\frac{1}{2} y\right) y
\end{array}\right.
$$

où la fonction $t \rightarrow u(t)$ est la fonction périodique définie par $u(t)=1$ si $2 k d t \leq t<$ $(2 k+1) d t ; \quad k=0,1, \ldots$ et $u(t)=0$ sinon. Ce sont donc les systèmes 1 puis 2 puis 1 puis 2 et ainsi de suite qui sont activés selon que $u$ vaut 0 ou 1 . Dans la simulation la constante $d t$ vaut 0,005 . On voit que, quelles que soient les conditions initiales, on assiste à la disparition de l'espèce $\mathrm{B}$. L'issue de la compétition est à l'opposée de celle observée dans un des deux environnements E I ou E II . Donc la succession périodique des deux environnements favorables à l'espèce B conduit à un environnement favorable à l'espèce A !

Nous venons de fournir un "contre-exemple" à l' affirmation 2.1. Nous pourrions en rester là mais, pour diverses raisons que je ne discute pas ici ${ }^{3}$, nous savons qu'il n'est pas sage de se servir de résultats de simulations quand ces dernières ne sont pas effectuées dans un cadre théorique suffisamment développé. C'est pourquoi nous théorisons un peu sur ce contre-example. 


\subsubsection{Une théorisation du "contre exemple"}

Un résultat du folklore de la théorie des systèmes ${ }^{4}$ affirme que si une suite $u_{n}$ de contrôles converge faiblement vers $u$ alors les trajectoires correspondantes convergent uniformément (sur les intervalles bornés) vers la trajectoire correspondant à $u$. Dans notre exemple $u$ est la fonction qui vaut $\frac{1}{2}$. Donc nous savons que si la fréquence des oscillations de $t \rightarrow u(t)$ est assez grande le système obtenu sera proche du système dont le second membre est la demi-somme des second membres de (2) et (3). Or il se trouve que cette demi-somme définit à son tour un système de compétition de Volterra. C'est le système :

$$
\left\{\begin{array}{l}
\frac{d x}{d t}=\frac{7}{4}\left(1-\frac{31}{8} x-\frac{31}{8} y\right) x \\
\frac{d y}{d t}=\frac{7}{4}\left(1-\frac{38}{8} x-\frac{38}{8} y\right) y
\end{array}\right.
$$

On voit que maintenant $a<c$ et $b<d$. Pour ce système, c'est A qui remporte la compétition. Ce calcul et sa conséquence, à savoir que, pour une fréquence suffisamment grande de passage d'un environnement à l'autre, c'est l'espèce A qui remporte la compétition sont incontestable. Donc, lorsqu'elle est assez rapide, la succession d'environnements E1, E2, E1, E2,.... est favorable à A ce qui est contraire à ce qu'affirme la proposition (informelle) (2.1) qui est donc invalidée par le contre-exemple.

Nous avons construit, à l'aide des équations de compétition de Volterra, un monde virtuel (mathématico-informatique) que nous reconnaissons comme illustrant correctement une situation de compétition. L'exemple proposé met en évidence que le concept "d'environnement favorable à une espèce" ne peut pas être manipulé de façon informelle comme il l'a été au début du chapitre pour conduire à la proposition (2.1). Nous devons soit renoncer à utiliser "environnement favorable à ..." ou bien lui donner un sens plus précis, ce qui passera par une plus grande formalisation du concept. A vrai dire un examen plus attentif d'un portrait de phase d'une équation de compétition de Volterra (voir figure (3)) nous éclaire un peu plus sur ce qui se passe : On voit que l'expression "environnement favorable" à B traduit une propriété asymptotique du système : Lorsque le temps tend vers l'infini $x(t)$ tend vers 0 alors que $y(t)$ tend vers une limite non nulle. En revanche nous voyons bien que pendant les transitoires il n'est pas possible de dire que l'environnement est "favorable à B" (ou A). Pour les trajectoires 1 et 2 les deux populations voient simultanément leur taille diminuer et, plus grave, pour la trajectoire 3 c'est la taille de la population A qui, pendant un certain temps, augmente le plus vite, laissant croire que l'environnement serait favorable à $\mathrm{A}$.

\subsubsection{Résumé de l'exemple 1}

Après avoir exposé au paragraphe 2.1.1 le contexte de ce que l'on pourrait appeler une loi en écologie, au paragraphe 2.1.2 j'énonce de façon informelle une loi dont la plausibilité semble grande. Je formalise la situation au paragraphe 2.1.3 et montre par un "contre exemple" au paragraphe 2.1.4 que la loi informelle n'est pas acceptable. Au paragraphe 2.1.5 une analyse mathématique du contre exemple permet d'aller plus loin dans la direction d'une reformulation plus satisfaisante de la "loi écologique" de départ".

4. voir, par exemple [5].

5. D'autres effets paradoxaux du même type que celui-ci sont décrits dans [6, 7]. 


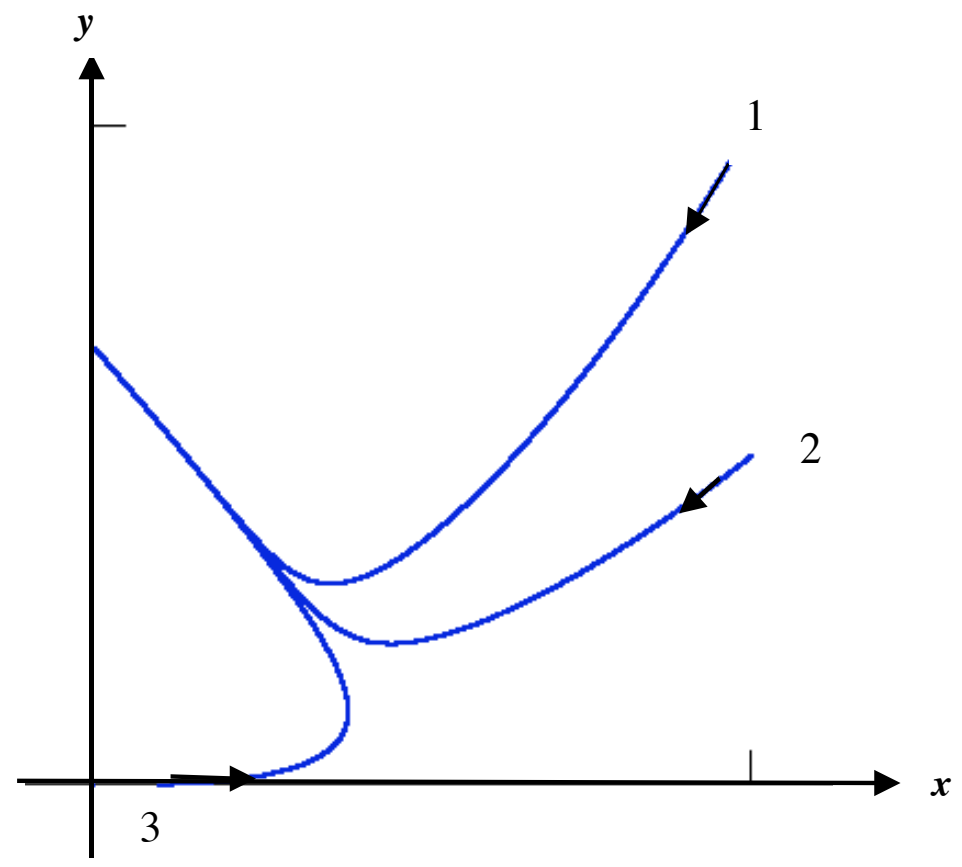

Figure 3. Portrait de phase d'une équation de compétition de Volterra

\subsection{Exemple 2 : La théorie des bifurcations dynamiques}

Au milieu des années 1980 et jusqu'en 1995 environ il y a eu une activité mathématique significative sur un thème qui est connu maintenant sous le nom de "Théorie des Bifurcations Dynamiques". Je développe dans cet exemple un moment de l'histoire de cette théorie ${ }^{6}$.

\subsubsection{Le contexte}

On considère une famille de systèmes différentiels :

$$
\frac{d x}{d t}=f(x, \mu)
$$

indexés par le paramètre $\mu$. On suppose que $\phi(\mu)$ est un équilibre, c'est à dire que pour tout $\mu$ l'on a :

$$
f(\phi(\mu), \mu)=0
$$

6. Pour un historique plus complet et des développements récents voir dans ce volume l'article de A. Fruchard et R. Schafke. 
On note $D f(\mu)$ la matrice jacobienne de $x \rightarrow f(x, \mu)$ au point $\phi(\mu)$ et on suppose qu'il existe une paire de valeurs propres conjuguées de $D f(\mu)$ notées $\alpha(\mu) \pm i \beta(\mu)$ dépendant continuement de $\mu$ telle que $\beta(0)>0$ et telle que :

$$
\begin{aligned}
& \mu<0 \Rightarrow \alpha(\mu)<0 \\
& \mu=0 \Rightarrow \alpha(\mu)=0 \\
& \mu>0 \Rightarrow \alpha(\mu)>0
\end{aligned}
$$

On suppose de plus que toutes les autres valeurs propres sont à parties réelles strictement négatives et que pour $\mu=0$ l'équilibre est asymptotiquement stable (mais pas exponentiellement stable !). On a alors le résultat suivant (Théorème de Poincaré, Andronov, Hopf) :

Théorème 2.2 (Poincaré, Andronov, Hopf) Sous les hypothèses ci-dessus, pour $\mu>0$ assez petit, le système (6) possède un cycle limite dont la taille (le diamètre) dépend continuement de $\mu$ et est de l'ordre de $\sqrt{\mu}$.

\subsubsection{Quelques commentaires classiques sur la bifurcation de Hopf}

Comme il est usuel, ce théorème est accompagné dans la littérature de "commentaires explicatifs". En voici trois.

Selon Marsden et McKraken.

Dand le livre de J.E. Marsden et M. McKraken The Hopf bifurcation and its applications, Springer Verlag, 1976, on lit page 9 :

The Hopf bifurcations refers to the development of periodic orbits ("self-oscillations") from a stable fixed point, as a parameter crosses the critical value.

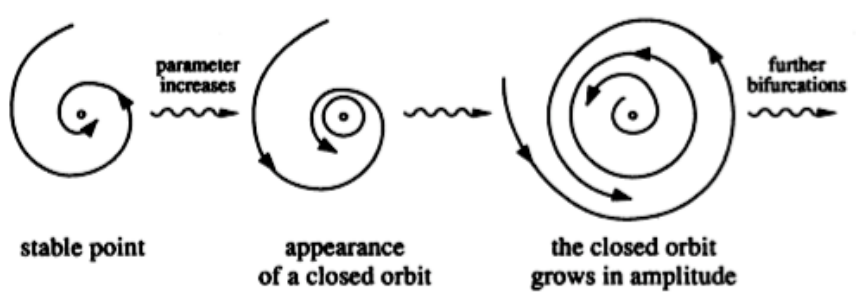

On voit que, dans ce commentaire, le paramètre est considéré comme une "variable dynamique" qui croit au cours du temps pour "traverser" la valeur critique 0.

\section{Selon V. Arnold}

Dans son célèbre cours Chapitres supplémentaires à la théorie des équation différentielles ordinaires , Editions Mir, Moscou 1980, V.I. Arnold commente ainsi : 
«Lorsque $\mu$ passe par 0 , le foyer de l'origine des coordonnées perd sa stabilité. Pour $\mu=0$, l'origine des coordonnées est également un foyer stable, mais non structurellement stable : les trajectoires ne se rapprochent pas exponentiellement de 0 .

Pour $\mu>0$ les trajectoires s'éloignent du foyer à une distance proportionnelle à $\sqrt{\mu}$ et s'enroulent autour d'un cycle limite stable. Donc, lorsque $\mu$ passe par 0 , la perte de stabilité s'accompagne de la naissance d'un cycle limite stable dont le rayon croit comme $\sqrt{\mu}$.

En d'autres termes, l'état stationnaire perd sa stabilité et il apparaît un régime périodique stable dont l'amplitude est proportionnelle à la

racine carrée de l'écart du paramètre par rapport à la valeur critique. Les physiciens parlent dans ce cas d'excitation douce d'auto-oscillations. ”

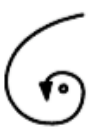

$\mu<0$

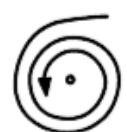

$\mu=0$

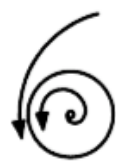

$\mu>0$

\title{
Toujours V. Arnold
}

Dans son livre Catastrophe theory, Springer Verlag, 1886 pp. 20-21, V. Arnold développe encore plus explicitement cette vision dynamique.

\begin{abstract}
A. On change of the parameter the equilibrium state gives birth to a limit cycle (radius of order $\sqrt{\varepsilon}$ where the parameter differs from the bifurcation value by $\varepsilon$ ). The stability of the equilibrium is transferred to the cycle, the equilibrium point itself become unstable.
\end{abstract}

This form of loss of stability is called 'seft' loss of stability since the oscillating state for small criticality differs little from the equilibrium state.

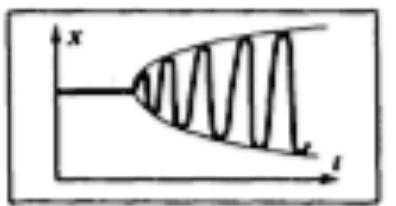

Fig 17

La figure qui accompagne le commentaire est très explicite : En même temps que le système évolue le paramètre change de valeur. 


\subsubsection{Commentaire sur ces trois commentaires}

Le théorème, dans sa version "formelle", dit que pour une valeur de $\mu$ strictement positive le système possède un cycle limite. Il ajoute que la dépendance de la taille du cycle par apport à $\mu$ est continue. Or la vision classique de la continuité est une vision dynamique dans laquelle les variables changent au cours du temps. D'où les commentaires naturels qui sont proposés : Quand $\mu$ croit (sous entendu au cours du temps) il se passe quelque chose. Ce "quelque chose" concerne les propriétés asymptotiques d'un système dynamique, donc des propriéttés où le temps est déjà impliqué. Mais le "temps du paramètre" et le temps du "système dynamique" sont ils les mêmes ?7. C'est sur ce point que les commentaires ne sont pas très clairs. Une façon très simple de clarifier la situation est de considérer les deux systèmes :

$$
\begin{aligned}
& \left\{\begin{array}{l}
\frac{d x}{d t}=f(x, \mu) \\
\frac{d \mu}{d t}=0
\end{array}\right. \\
& \left\{\begin{array}{l}
\frac{d x}{d t}=f(x, \mu) \\
\frac{d \mu}{d t}=\varepsilon
\end{array}\right.
\end{aligned}
$$

où $\varepsilon$ est un paramètre strictement positif. Dans le premier cas le paramètre $\mu$ est constant. Pour chaque valeur initiale de $\mu$ le système évolue selon les prédiction du théorème 2.2. Pour $\varepsilon>0$ le système (8) est une "perturbation singulière" (singulière parce que les propriétés considérés sont asymptotiques) de (7). Le commentaire sera donc correct si nous sommes capables de montrer que pour $\varepsilon$ assez petit les propriétés de (8) sont les mêmes que (ou au moins sont proches de) celles de (7). Or il n'en est rien.

\subsubsection{Un contre exemple à l'interprétation dynamique}

Soit le système :

$$
\left\{\begin{aligned}
\frac{d x_{1}}{d t} & =x_{2}+\mu x_{1}-x_{1}\left(x_{1}^{2}+x_{2}^{2}\right) \\
\frac{d x_{2}}{d t} & =-x_{1}+\mu x_{2}-x_{1}\left(x_{1}^{2}+x_{2}^{2}\right) \\
\frac{d \mu}{d t} & =\varepsilon
\end{aligned}\right.
$$

où, en coordonnées polaires, ce qui est peut être plus clair :

$$
\left\{\begin{array}{l}
\frac{d \theta}{d t}=1 \\
\frac{d \rho}{d t}=\rho\left(\mu-\rho^{2}\right) \\
\frac{d \mu}{d t}=\varepsilon
\end{array}\right.
$$

La figure 4, extraite de [8], représente une simulation de l'intégration de (9) avec $\mu(t)$ en abcisse et $\rho(t)$ en ordonnée à partir de la condition initiale $(0,1,-2)$ avec $\varepsilon=0.01$. On ne reconnaît pas la transition progressive d'un état stationnaire stable vers un cycle

7. L'analyse Non Standard, quoi qu'on pense par ailleurs de sa pertinence dans la pratique mathématique, permet de bien voir sur cette question de la continuité comment les mathématiques ne se réduisent pas à un simple jeu formel. Je développe ce point plus loin dans lee paragraphe 3.3.3) 


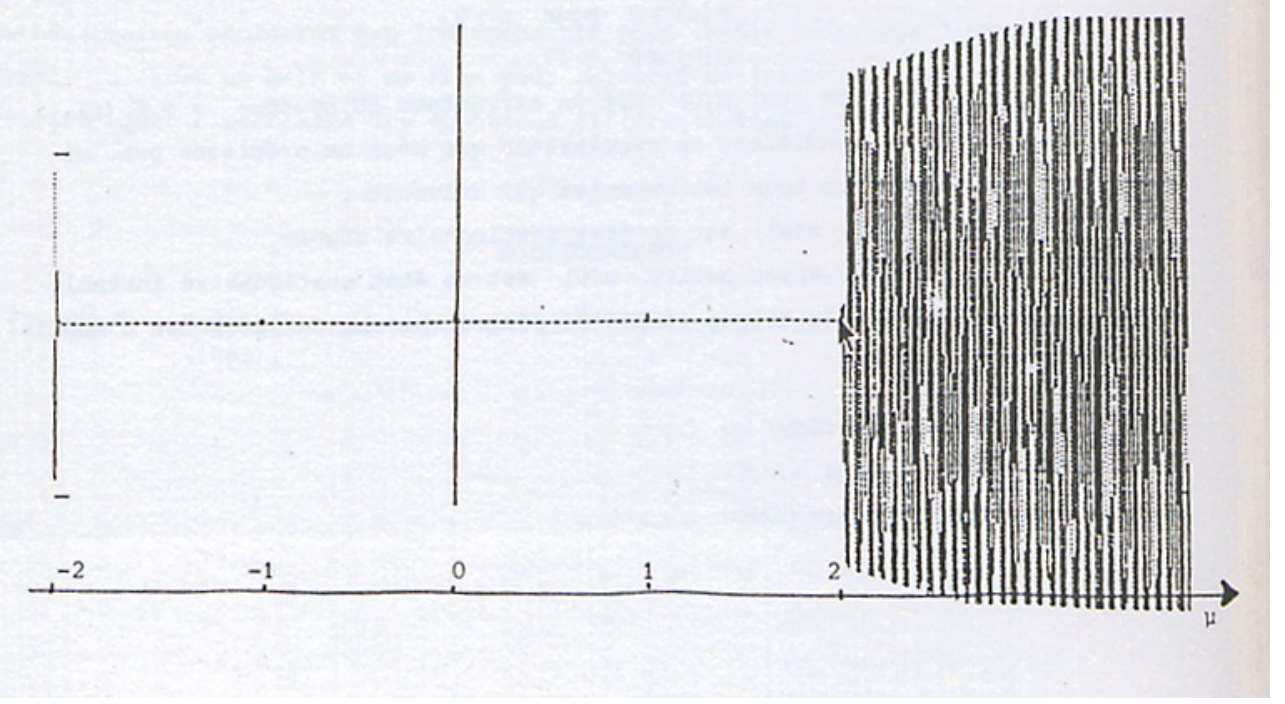

Figure 4. Simulation de 10, le temps est en abcisse, $\rho$ en ordonnée.

limite de plus en plus grand prévue dans les commentaires. Toutefois cela ne suffit pas à les disqualifier complètement pour au moins deux raisons. La première est que le "contre exemple" est un peu particulier : il se pourrait que la symétrie parfaite de la rotation soit à l'origine d'un fait exceptionnel. La seconde est que, dans une application au monde réel, la présence de bruit ne devrait pas permettre de se maintenir sur l'équilibre instable. La réponse à ces questions et à d'autres se trouve dans la téorie des bifurcation dynamiques dont je cite un des résultats majeur au paragraphe suivant

\subsubsection{Le théorème de Shishkova, Neishtadt, Callot}

On considère le système (8) ci-dessus où la famille $f(x, \mu)$ satisfait les hypothèses du thérorème 2.2 et où $\varepsilon$ est infiniment petit au sens de l'Analyse Non Standard ${ }^{8}$.

Théorème 2.3 Si le second membre de (8) est analytique et seulement dans ce cas, soit une condition initiale $\left(x_{0}, \mu_{0}\right)$ avec $\mu_{0}<0$ et non infiniment proche de 0 . Dès que t est infiniment grand et tant que $\mu(t)$ reste plus petit qu'une certaine quantité $\sigma\left(\mu_{0}\right)$ le point $x(t)$ reste infiniment proche de $\psi(t)$. La quantité $\sigma\left(\mu_{0}\right)$ est une fonction croissante de la valeur absolue de $\mu_{0}$ et est non infiniment petite dès que cette dernière l'est.

C'est ce phénomène qui est traduit par l'expression retard à la bifurcation. La bifurcation ne se produit pas en 0 où elle était attendue, mais plus tard.

8. les termes écrits en gras doivent être entendus au sens (formel) de l' A.N.S. 
L'objection concernant le bruit possède une réponse nuancée. Tout dépend du type de "bruit". Si le bruit est "additif", c'est à dire de la forme :

$$
\frac{d x}{d t}=f(x, \mu)+\frac{d B}{d t}
$$

où $\frac{d B}{d t}$ est un bruit blanc gaussien (selon la notation des ingénieurs) il y a effectivement disparition du phénomène de retard. Mais, au contraire, si le bruit est multiplicatif , c'est à dire de la forme :

$$
\frac{d x}{d t}=f(x, \mu)+x \frac{d B}{d t}
$$

alors le phénomène de retard est accentué (voir par exemple $[9,10])^{9}$.

\subsubsection{Résumé de l'exemple 2}

Après avoir exposé au paragraphe 2.2.1 le contexte de la théorie des bifurcation des systèmes dynamiques, au paragraphe 2.2.2 j'énonce de façon informelle quelques commentaires du théorème de Poincaré, Andronov, Hopf. Je formalise les doutes nés d'une lecture attentive des commentaires au paragraphe 2.2 .3 et montre par un contre exemple au paragraphe 2.2.4 que le "commentaire formalisé" est faux et donc que les commentaires ne sont pas acceptables en l'état. Au paragraphe 2.2.5 j'énonce quelques résultats issus de la considération du nouveau problème formalisé qui donnent un aperçu de la théorie qui se développe à partir de la nouvelle formalisation.

\section{La méthode des élucidations successives}

Avant de commencer ce paragraphe il peut être utile de rappeler les résumés (2.1.6) et (2.2.6) des exemples 1 et 2.

\subsection{Comparaison des exemples 1 et 2}

Exemple 1. Après avoir exposé au paragraphe 2.1.1 le contexte de ce que l'on pourrait appeler une loi en écologie, au paragraphe 2.1.2 j'énonce de façon informelle une loi dont la plausibilité semble grande. Je formalise la situation au paragraphe 2.1.3 et montre par un "contre exemple" au paragraphe 2.1.4 que la loi informelle n'est pas acceptable. Au paragraphe 2.1.5 une analyse mathématique du contre exemple permet d'aller plus loin dans la direction d'une reformulation plus satisfaisante de la "loi écologique".

Exemple 2. Après avoir exposé au paragraphe 2.2.1 le contexte de la théorie des bifurcation des systèmes dynamiques, au paragraphe 2.2.2 j'énonce de façon informelle quelques commentaires du théorème de Poincaré, Andronov, Hopf. Je formalise les doutes

9. Le théorème (au sens formel) (2.2.5) a été énonçé et démontré en 1973 pour la première fois par Shiskova dans une version simplifiée sous la forme d'une courte note [11] sans commentaire. Cette note est passée relativement inaperçue, de même que les articles de Haberman [12] en 1976 et Rubenfeld [13] en 1979 qui, eux, posaient bien la problématique du retard, mais en dimension 1. A peut près simultannément et indépendamment, plusieurs auteurs $([8,14,15,16,17])$ ont abordé le thème de ce paragraphe qui a été largement développé un peu plus tard dans l'ouvrage collectif [18]. L'esprit du travail de Shiskova : travailler avec un "temps complexe", devait réapparaître en 1991 puis 1993 chez Wallet ([19]) puis Callot ([20]) associé à de l'ANS. 
nés d'une lecture attentive des commentaires au paragraphe 2.2.3 et montre par un "contreexemple" au paragraphe 2.2.4 que le "commentaire formalisé" est faux et donc que les commentaires ne sont pas acceptables en l'état. Au paragraphe 2.2.5 j'énonce quelques résultats issus de la considération du nouveau problème formalisé qui donnent un aperçu de la théorie qui se développe à partir de la nouvelle formalisation.

Ce qui peut donner, dans le plus pur style "bourbachique" :

Après avoir exposé au paragraphe 2.1.1 (resp.2.2.1) le contexte de ce que l'on pourrait appeler une loi en écologie (resp. le contexte de la théorie des bifurcation des systèmes dynamiques) au paragraphe 2.1.2 (resp.2.2.2) j'énonce de façon informelle une loi dont la plausibilité semble grande. (resp. quelques commentaires sur le théorème de Poincaré, Andronov, Hopf.) Je formalise la situation (resp. les doutes nés d'une lecture attentive des commentaires ) au paragraphe 2.1.3 (resp. 2.2.3) et montre par un "contre-exemple" au paragraphe 2.1.4 (resp. 2.2.4) que la loi informelle n'est pas acceptable. (resp. que le "commentaire formalisé" est faux et donc que les commentaires ne sont pas acceptables en l'état.) Au paragraphe 2.1.5 (resp. 2.2.5) une analyse mathématique du contre-exemple permet d'aller plus loin dans la direction d'une reformulation plus satisfaisante de la "loi écologique" (resp. j'énonce quelques résultats issus de la considération du nouveau problème formalisé qui donnent un aperçu de la théorie qui se développe à partir de la nouvelle formalisation.).

Dans un style plus léger nous dirons qu'une situation d'un contexte donné est l'objet d'une interprétation-1 qui pose problème; l'interprétation est formalisée dans un texte mathématique qui est traité selon les techniques du calcul et de la démonstration et les résultats obtenus font à nouveau l'objet d'une interprétation-2, ce que je résume dans le schéma : ......

Interprétation- $1 \rightarrow$ Formalisation-1

Formalisation-1 + Calcul-démonstration $\rightarrow$ Résultat formel-2

Formalisation-2 $\rightarrow$ Intreprétation-2

$\cdots \cdots$

Ce qui fait la différence entre mes deux exemple ce n'est donc pas la méthode, mais le contexte, l'objet de l'utilisation de la méthode. Avant d'aller plus loin j'essaye de caractériser mieux la méthode en question.

\subsection{La méthode des élucidations successives.}

En mathématiques élémentaires, une méthode d'approximations successives est un algorithme qui permet de donner des valeurs successives de plus en plus précises d'une racine d'une équation. Ainsi la suite :

$$
x_{n+1}=\frac{2+x_{n}^{2}}{2 x_{n}} ; x_{1}=1
$$

donne-elle des valeurs de plus en plus précises de $\sqrt{2}$, en d'autres termes la distance qui sépare $x_{n}$ de $\sqrt{2}$ est de plus en plus petite. Il est tentant de voir dans la succession :

Interprétation- $\mathrm{n} \rightarrow$ Formalisation- $\mathrm{n}$

Formalisation- $\mathrm{n}+$ Calcul-démonstration $\rightarrow$ Résultat formel- $\mathrm{n}+1$ 
Formalisation- $n+1 \rightarrow$ Intreprétation-n+1

.......

une étape d'un algorithme qui nous permettrait de nous approcher progressivement de l'interprétation parfaite, c'est à dire du "Sens" ou de la "Vérité". Je ne souhaite pas retenir cette vision d'une "progression" vers le "Sens". En effet la comparaison avec la méthode des approximations successives nous rappelle aussi que la nature de 1" "existence" de ce dont on se rapproche peut poser problème. Qu'est ce que $\sqrt{2}$ ? Officiellement, pour les théories mathématiques classiques (standard), $\sqrt{2}$ est une "classe d'équivalence de suites de Cauchy" particulière, un nombre "réel particulier"; il existe des règles de calcul qui régissent l'emploi des nombres réels et il suffit de vérifier en respectant ces règles que la suite définie par (11) se rapproche effectivement de $\sqrt{2}$. Mais la question de la "nature en soi" de $\sqrt{2}$ reste une question philosophique qui est toujours loin d'être tranchée. Ainsi, on conçoit que si quelque chose d'aussi simple que $\sqrt{2}$ pose problème, alors ce qu'est le fameux "Sens" dont notre méthode des élucidations successives permettrait de se rapprocher en pose un plus formidable encore ${ }^{10} 11$.

C'est pourquoi, pour affiner la métaphore, je propose de reconsidérer (11) non comme une suite qui tend vers $\sqrt{2}$ mais comme une suite dont les carrés tendent vers 2 . De la même manière que l'algorithme défini par (11) définit une suite de nombres $x_{n}$ de plus en plus satisfaisants (du point de vue de leur carré) sans qu'il soit besoin d'invoquer un nombre dont le statut ontologique pose problème, les élucidations successives peuvent très bien être comprises comme des textes de plus en plus satisfaisants sans qu'il soit nécessaire d'invoquer une convergence vers une "chose" qui serait "le Sens" ou "la Vérité". Bien entendu j'ai déplacé la difficulté sur "satisfaisant". Je propose de la résoudre en prenant cette expression au sens empirique : le nouvel énoncé est plus "satisfaisant" si la communauté des spécialistes en décide ainsi. De toutes façons, il faut remarquer que les choix de chacun sur le mode d'existence du "Sens" ou de la "Vérité" ne change pas sa pratique des élucidations successives.

En plus du caractère itératif de la méthode je retiens le mode très particulier du passage d'un texte au suivant, à savoir, l'usage d'un "modèle formel" (mathématique) du texte $n$ formalisation qui permet de produire des conséquences logiques du "modèle". Dans le premier exemple le "modèle" est un système d'équations différentielles, dans le second cas le modèle est dans le couple de systèmes différentiels l'un avec $\mu^{\prime}=0$ l'autre avec $\mu^{\prime}=\varepsilon$. Dans les deux cas il est essentiel que l'accord se fasse sur le fait que le modèle proposé est pertinent. C'est à cette condition seulement que nous prendrons au sérieux les prédictions du modèle. En revanche, une fois le modèle accepté, il n'y a aucun doute sur l'exactitude des prédictions puisqu'elles relèvent de la "démonstration mathématique". En d'autres termes, dans la méthode des élucidations successives, aucun doute ne saurait entacher l'étape :

10. Toujours sur la question de la "convergence" des élucidations successives vers quelque chôse". Un ami, Pierre Bernhard, qui avait assisté à une de mes conférences sur ce thème ne se souvenait plus si j'avais employé "progressives" ou "successives". A premère vue je n'y voyais pas une grande différence, puis, en y réfléchissant, je me suis fixé sur "successives". En effet, dans "progressive" il y a l'idée que l'on progresse vers quelque chose. Ce que je veux éviter. 11. Dans [21], J. Harthong expose comment on pourrait développer une analyse qui se "contenterait" du corps des rationnels, sans avoir besoin de le compléter. 
Formalisation-n + Calcul-démonstration $\rightarrow$ Résultat formel-n+1

car si doute il y avait cela reviendrait à dire que le texte de l'étape $n$ n'a pas été suffisamment formalisée.

\section{Commentaires sur la Méthode des Elucidations Successives}

Je ne crois pas avoir inventé grand chose mais, si j'ai lu divers textes qui m'ont inspiré, je n'en ai pas rencontré qui dise exactement ce que je viens de dire. Voici donc mes sources et en quoi j'y reconnais les élucidation successives.

La première source est le livre de I. Lakatos "Preuves et Réfutations", la seconde est le livre de J-M. Salanskis "L'Herméneutique formelle" et enfin, la dernière est un article de W.O. Quine "Deux dogmes de l'empirisme". Je terminerai ces commentaires par un point de vue du biologiste A. Danchin.

\subsection{La logique de la découverte mathématique selon I. Lakatos}

Dans son livre ${ }^{12}$ Lakatos s'en prend à ce qu'il appelle l'école formaliste qu'il définit comme l'école de philosophie mathématique qui tend à identifier les mathématiques à leur abstraction axiomatique formelle et qui oublie la construction historique de la mathématique vivante. Au contraire il soutient que les mathématiques ont une histoire et que ses résultas se construisent par des conjectures successives qui sont régulièrement infirmées par des contre exemples qui permettent de reformuler la conjecture initiale et ainsi de suite, je cite :

Son modeste projet (la présente étude) est d'étudier en détail la thèse suivant laquelle les mathématiques non formelles, quasi empiriques, ne se développent pas dans un accroissement continu du nombre des théorèmes indubitablement établis, mais dans l'amélioration constante des conjectures grâce à la spéculation et à la critique, grace à la logique des preuves et réfutations.

Il étaye sa thèse par une minutieuse analyse de l'histoire de la formule d'Euler :

$$
S-A+F=2
$$

selon laquelle, dans un polyèdre, le nombre des sommets moins le nombre des arrêtes plus le nombre des faces est toujours égal à deux. Il fait reconstruire à une classe imaginaire, dans une sorte de dialogue Socratique, l'histoire de cette conjecture qui démarre en 1630 avec Descartes et se termine dans la seconde moitié du vingtième siècle par la topologie algébrique moderne en passant par Euler et Poincaré pour ne citer que les plus célèbres. Il n'est pas question de résumer en quelques lignes 160 pages d'une exceptionnelle finesse. Je me contente de reproduire la "méthode de preuve et réfutation" telle qu'énoncée par l'élève Lambda:

12. qui est tiré de sa thèse [22]. 
Règle 1: En présence d'une conjecture, mettre en chantier sa preuve comme sa réfutation. Examiner la preuve avec précaution pour préparer une liste de lemmes non triviaux (la preuve-analytique); trouver des contre-exemples à la fois à la conjecture (aspect global) et aux lemmes suspects (aspect local).

Règle 2 : En présence d'un contre-exemple global, écarter votre conjecture, ajouter à la preuve-analytique un lemme convenable, réfuté par le contre-exemple, et remplacer la conjecture écartée par une version améliorée en lui incorporant ce lemme sous forme de condition. ${ }^{69}$ Ne pas accepter qu'une réfutation soit écartée comme un monstre. ${ }^{70}$ Essayer d'expliciter tous les 'lemmes cachés'. ${ }^{71}$

Règle 3: En présence d'un contre-exemple local, vérifier s'il n'est pas non plus global. Si oui, il est aisé d'appliquer la Règle 2.

qui montre que nous ne sommes pas loin des élucidations successives sauf peut être que le côté itératif n'est pas mis en évidence (mais il est sous entendu dans tout l'essai) et que la méthode est présentée comme interne aux mathématiques. C'est sur ce dernier point que j'attire l'attention. J'ai voulu montrer, par mon exemple 1 que la méthode n'est pas propre aux mathématiques.

\subsection{L'herméneutique formelle de J-M Salanskis}

Commençons par une citation qui montrera clairement mon emprunt. Dans livre de J-M Salanskis Le Temps du Sens [23], qui est postérieur à L'Herméneutique Formelle [24] (un essai sur la présence de la démarche herméneutique au sein des mathématiques), on trouve le shéma :

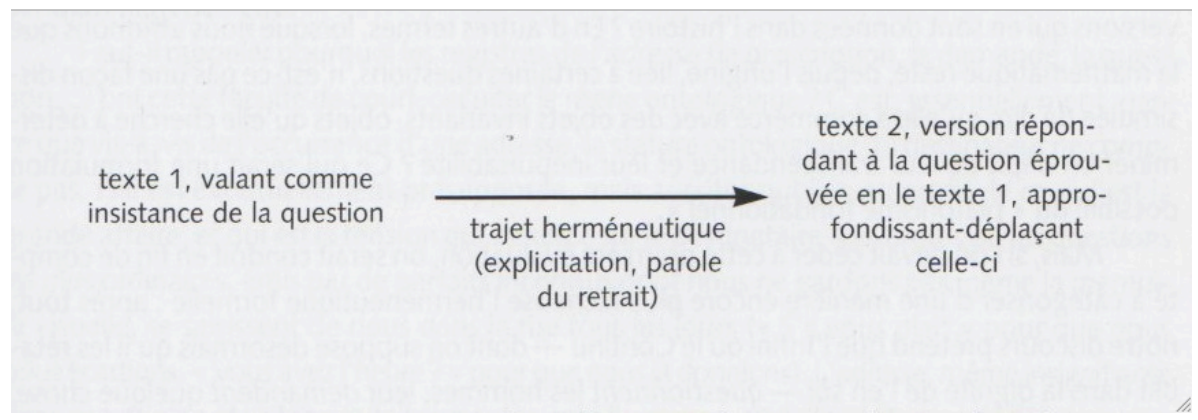

présenté comme l'essence de la démarche herméneutique. D'autre part l'ouvrage collectif L'heméneutique : textes, science [25] édité par J-M. Salanskis et des collègues est consacré à la démonstration de la présence de la démarche herméneutique dans les sciences en géneral. On peut donc considérer que les élucidations successives, sinon telles que je les décrits, au moins dans l'esprit, sont présentes dans les travaux de J-M Salanskis. Pour enfoncer le clou encore une citation, extraite de L'Herméneutique Formelle qui montre que je n'ai même pas introduit "élucider". 
Très grossièrement, on pourrait dire que la poésie élucide de mot en mot, que la philosophie élucide de mot à système, et que la mathématique élucide de système à système. Le trajet poétique est une pure dérive le long du lexique de la ou des langues habitées par le poème, et parmi les champs de force qui hantent celles-ci ; le trajet philosophique est une installation de connexions, d'ordre, de valeur signifiante, à partir de la sollicitation infinie qui émane de certains mots ; cette installation a comme opérateur privilégié, à côté de la logique générale, la

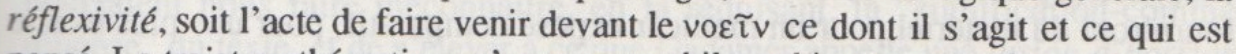
pensé. Le trajet mathématique s'oppose au philosophique comme le poétique, en ce qu'il est une "dérive" non réflexive (la déduction est une dérive, on dit dérivation formelle de nos jours), mais il se distingue du poétique en ce qu'il est une dérive dans le rapport plutôt que dans l'élément lexical, le passage est toujours passage d'une syntaxe, d'une cohérence, d'un système à un autre, passage qui s'accomplit, s'anticipe dans et se laisse guider par l'opacité du calcul.

\subsection{W. V. O. Quine et les vertus du double langage.}

Si nous reprenons l'exemple 1 nous avons une phrase : Une succession d'environnements favorables à l'espèce A est favorable à l'espèce A, écrite dans la langue naturelle, les termes environnement, espèce et favorable ayant été explicités préalablement dans la desciption du contexte. Cette phrase est traduite en un texte ${ }^{13}$ écrit dans une langue beaucoup plus formelle, mais qui est sensée représenter la même situation.

Dans l'exemple 2 nous avons un commentaire du genre : Lorsque $\mu$ passe par la valeur 0 il y a apparition d'un cycle limite dont l'amplitude croit comme $\sqrt{\mu}$, qui est clairement écrit dans une langue mathématique, mais une langue assez "relachée" et le commentaire est traduit en un texte beaucoup plus formel ${ }^{14}$ qui est sensé modéliser notre commentaire.

La question se pose de savoir pourquoi nous passons notre temps à écrire des formulations vagues, imprécises, ambiguües et à les traduire ? Ne serait-t-il pas raisonnable d'écrire "une fois pour toutes", dès le début, des formulations précises et sans ambiguité ? C'est le vieux rêve formaliste : reprendre tout à zéro sur une base sure ${ }^{15}$ et développer rigoureusement, rêve initial de Bourbaki. Le problème est que l'esprit humain ne fonctionne pas ainsi. Lorsqu'une formulation est trop précise elle devient très longue, très, très longue, si longue que lorsqu'on arrive à la fin on en a oublié le début. Il est nécessaire d'utiliser

13. Ici le texte formel est un système d'équations différentielles, donc écrit dans la "langue mathématique" que je considère comme "incluse dans la lague naturelle.

14. Ici encore un système d'équations différentielles; dans ce système toute la nuance entre savoir si $\mu$ "varie" dans un temps qui est celui des systèmes dynamiques ou un "autre temps", nuance que je trouve assez difficile à exprimer dans la langue naturelle, est traduite de façon incisive par la différence entre $\mu^{\prime}=0$ et $\mu^{\prime}=\varepsilon$.

15. Ici il faut rappeler ce qu'est le "Bateau" de Neurath. O. Neurath était un membre du Cercle de Vienne qui a proposé la métaphore suivante : Nos connaissances sont comme un bateau (sur lequel nous sommes embarqués) dont nous constatons en permanence qu'il est mal conçu au départ. II faudrait tout reconstruire, reprendre à zéro. Le problème est que ce bateau navigue sur un océan où il n'y a pas de continent, ni d'ile, ni à plus forte raison de port. II n'y a d'autre solution que de rafistoler, planche par planche, en faisant attention qu'une amélioration locale ne compromette pas l'ensemble. 
des mots qui recouvrent tout un discours au prix d'une certaine imprécision quitte à revenir sur une formulation plus précise. Cette navigation à l'intérieur de la langue entre des concepts plus ou moins précis se fait le plus souvent de façon graduelle, continue. Ce que j'essaye de montrer, dans mes deux exemples, c'est comment la "modélisation", c'est-à-dire l'écriture de systèmes d'équations mathématiques, est un cas particulièrement extrême de ce travail sur la langue : d'un côté la langue naturelle de l'autre un système d'équations, pour dire la même chose.

Cette idée de la pratique simultanée de deux langues pour exprimer la même chose, cette idée d'une sorte de double langage est exprimée par Quine de façon lumineuse dans l'article "Deux dogmes de l'empirisme", repris dans "From the logical point of view", 1953 (bas de la page 56 de la traduction française, "Du point de vue logique", Vrin 2003).

Dans les systèmes logiques et mathématiques, on peut rechercher deux types d'économie possibles, mutuellement antagonistes; l'un et l'autre ont une utilité pratique particulière. D'une part on peut rechercher l'économie d'expression - on cherche alors à énoncer avec aisance et brièveté des relations aux multiples facettes. Ce type d'économie exige d'ordinaire des notations concises et caractéristiques pour des concepts abondants. D'autre part et par contraste, on peut chercher à économiser la grammaire et le vocabulaire. On peut essayer de trouver un minimum de concepts de base, tels que une fois qu'on a trouvé pour chacun une notation caractéristique, on puisse exprimer n'importe quel concept supplémentaire en combinant et en répétant simplement nos notations de base. En un sens, ce deuxième type d'économie n'est pas très pratique, puisque la pauvreté des expressions de base contribue nécessairement à rallonger le discours. Mais en un autre sens, il est pratique : il simplifie beaucoup le discours à propos du langage en minimisant les termes et les formes de construction dont se compose le langage.

Même si elles sont à première vue incompatibles, ces deux espèces d'économie sont précieuses à leur manière. Il est donc devenu courant de les combiner en forgeant de fait deux langages, dont l'un est une partie de l'autre. Le langage incluant sera redondant par sa grammaire et son vocabulaire, mais économique par la brièveté des ses messages. Le langage inclus, appelé notation primitive, sera économique par sa grammaire et son vocabulaire. Le tout et la partie sont corrélés par des règles de traduction grâce auxquelles chaque idiome, qui n'est pas écrit en notation primitive, est identifié à un complexe construit à partir de la notation primitive. Ces règles de traduction sont ce qu'il est convenu d'appeler des définitions, dans les systèmes formalisés. Il faut se les représenter, non pas comme des ajouts à un langage, mais comme des corrélation entre deux langages dont l'un est partie de l'autre.

L'Analyse Non Standard fournit une illustration de ce propos. le système formel I.S.T. de Nelson s'obtient à partir du système formel Z.F.C. par adjonction d'un prédicat à une place $s t(x)$ qui se lit $x$ est standard et à partir duquel il est possible de dériver des expressions comme "infiniment petit" qui seront utiles en analyse. I.S.T. est dans ce cas la langue incluante et il est connu que les expressions en Analyse Non Standard sont généralement plus concises et plus élégantes. Mais le prix à payer est un discours sur le concept $s t(x)$, discours que certains ont de la difficulté à accepter.

Mais l'Analyse Non Standard est aussi un exemple de ce que les mathématiques ne s'identifient pas à un système formel de référence. 
Ainsi considérons les deux couples :

Langage de la mathématique classique $\supset$ Langage formel Z.F.C.

Langage de l'Analyse Non Standard $\supset$ Langage formel I.S.T.

On sait que les langages Z.F.C. et I.S.T. sont logiquement équivalents. Mais les jeux "formalisation-interprétation", eux, sont différents. Voyons ce qu'il en est à propos de la notion de continuité.

La définition formelle classique de la continuité au point $x_{0}$ d'une fonction $x \rightarrow f(x)$ est :

$$
\forall \varepsilon>0 \exists \eta \forall h\left(|h|<\eta \Rightarrow\left|f\left(x_{0}+h\right)-f\left(x_{0}\right)\right|<\varepsilon\right)
$$

qui est traduite de diverses manières :

- quand $h$ tend vers $0 f\left(x_{0}+h\right)$, tend vers $f\left(x_{0}\right)$

- plus $h$ est petit, plus $\left.f\left(x_{0}\right)+h\right)$ est proche de $f\left(x_{0}\right)$

$-f\left(x_{0}+h\right)-f\left(x_{0}\right)$ peut être rendu aussi petit que l'on veut pourvu que $h$ soit suffisamment petit

Dans ces traductions il y l'idée que la variable $x$ est une position (un curseur sur un axe) pouvant varier de façon continue (pris au sens physique, c'est-à-dire capable d'occuper toutes les positions intermédiaires) et la valeur correspondante de la fonction varie à son tour. L'expression isolée "quand $x$ tend ver $x_{0}$ " n'a pas de traduction formelle. En d'autres termes la prhrase :

$$
\text { quand } h \text { tend vers } 0 f\left(x_{0}+h\right) \text {, tend vers } f\left(x_{0}\right)
$$

ne peut pas être coupée en morceaux si nous voulons qu'elle ait une traduction formelle immédiate. Il n'en reste pas moins que " $h$ tend vers 0 " a un sens non formel qui est "cinématique"; $h$ est une quantité qui varie au cours du temps et éventuellement se rapproche de 0 . La formulation classique de la continuité est une formulation "dynamique".

La formulation ANS de la continuité est dans le formalisme I.S.T. :

$$
\forall h\left[\left(\forall^{s t} a>0|h|<a\right) \Rightarrow\left(\forall^{s t} a>0\left|f\left(x_{0}+h\right)-f\left(x_{0}\right)\right|<a\right)\right]
$$

et est traduite par :

$$
\text { pour tout } h \text { infiniment petit } f\left(x_{0}+h\right)-f\left(x_{0}\right) \text { est infiniment petit }
$$

où, on le voit, " $u$ infiniment petit" est l'interprétation de :

$$
\left(\forall^{s t} a>0|u|<a\right)
$$

Dans la phrase pour tout $h$ infiniment petit $f\left(x_{0}+h\right)-f\left(x_{0}\right)$ est infiniment petit on peut isoler les morceaux $\{h$ infiniment petit $\}$ et $\left\{f\left(x_{0}+h\right)-f\left(x_{0}\right)\right\}$ est infiniment petit qui conservent une traduction formelle. Cette vision de la continuité est "statique".

Donc deux systèmes formels logiquement équivalent peuvent induire à travers le processus d'interprétation des langages mathématiques - et donc des pratiques mathématiques - 
très différents.

Je pense que ce que Quine dit des systèmes logiques et mathématiques a une porté plus large et est aussi à l'oeuvre dans la méthode des élucidations successives.

\subsection{La méthode générative critique de A. Danchin.}

Dans les actes [26] du colloque Elaboration et justification des modèles organisé en 1979 par P. Delattre et M. Thellier le biologiste A. Danchin développe ce qu'il appelle la méthode générative critique qu'il attribue à Xénophane. Je cite :

Les principes (admis sans discussion, mais, nous verrons comment, soumis à la critique) sont les suivants :

- (i) il existe une vérité, non contradictoire ;

- (ii) la vérité ne parle pas (elle n'est pas révélée).

Le principe (i) exclut le relativisme fort à la mode dans une partie de l'opinion qui veut que la connaissance ne soit qu'une image de la culture et de l'idéologie, sans aucun rapport avec le monde réel (lui même nié dans les formes les plus outrancières de ce relativisme). Je ne discuterai pas ici ....

.... admettant (ii) il est possible d'approcher de la vérité.

En effet, en vertu de l'existence d'une vérité il est possible de faire un pari sur ce qu'elle est. Evidemment, ce pari est presque à coup sûr dans l'inexactitude, mais ce que remarque Xénophane, c'est qu'il permet cependant de servir de point de départ d'une recherche de la vérité. On opère de la façon suivante : (souligné par moi) si le pari (ou la devinette) possède quelque élément d'inexactitude, il doit être possible de le réfuter par quelque particularité du monde réel. Dès que cela est obtenu un élément initial de la devinette est immanquablement repéré comme faux, il doit donc être remplacé par un autre élément, et l'on applique le même critère à la nouvelle devinette, et ainsi de suite. Comme on le voit, la méthode est générative et permet donc d'approcher par essais successifs, du vrai.

L'analogie est évidente avec la méthode des élucidations successives : processus itératif et sans fin. Toutefois je voudrais noter deux différences, l'une essentielle, l'autre secondaire.

L'essentielle pour commencer : la confrontation au monde réel. A aucun moment, dans le processus de clarification que je discute, il n'y a comparution devant le tribunal de la réalité. Je ne proclame pas qu'à l'étape $n+1$ le discours (le modèle) est plus proche de la "vérité"; je dis simplement qu'il est plus cohérent. Le juge du modèle $n$ n'est pas la "réalité" mais la "logique formelle" à l'oeuvre dans la démonstration mathématique. Même si l'on veut voir dans les contraintes de la logique une manifestation de la réalité on m'accordera, je pense, qu'il s'agit d'une réalité d'une autre nature que celle dont parle A. Danchin. Donc, dans les élucidations successives ce qui change ce n'est pas l'adéquation du discours à la réalité mais sa cohérence interne. J'insiste sur ce point car je crois que, dans la pratique effective de la méthode expérimentale, lors de la remise en cause du modèle $n$ et de son remplacement par le mpdèle $n+1$ ce sont souvent deux opérations qui sont menées de front : en priorité une confrontation à la réalité mais aussi clarification du discours. 
La seconde différence est secondaire pour ce que je discute ici. C'est, bien entendu, celle qui est relative à l'existence d'une "Vérité". On a vu que j'ai des réticences à parler de Vérité avec un grand $\mathrm{V}$, sans pour autant accepter le relativisme que dénonce Danchin. Mais c'est une autre discussion.

\section{Conclusion}

En résumé.

- Les élucidations successives sont le processus de preuves et réfutations décrit par I. Lakatos pour les mathématiques (et que l'on peut observer dans mon exemple 2) mais aussi à l'oeuvre en dehors du champ des mathématiques, notamment dans ce qu'on appelle la "modélisation" comme le montre mon exemple 1.

- Les élucidations successives ont quelque chose à voir avec l'herméneutique.

- Les élucidations successives ne doivent pas être confondues avec la démarche expérimentale de confrontations successives de plus en plus exigeantes avec la réalité.

Je me suis livré dans cet article, sur le texte que je proposais en introduction comme définissant la méthodes des élucidations successives et que je rappelle ci-dessous :

1) ...un texte numéro $n$ est écrit, interprété et commenté.

2) Les commentaires soulèvent des questions, des doutes sur l'interprétation du texte numéro $n$ voire le texte lui même.

3) Les commentaires et les doutes sont formalisés et l'on fait fonctionner la mécanique formelle pour en déduire.....

4) ....un texte $n+1$ et ses commentaires qui clarifient le texte $n$ et ses commentaires mais soulèvent à leur tour des questions....

à la phase 1 d'une itération : j'ai interprété et commenté. Maintenant, des doutes doivent normalement s'élever à propos de ce texte. En voici quelques un ${ }^{16}$ :

On peut se demander s'il y a quelque chose de spécifique à la modélisation dans les élucidations successives comme le laisse plus ou moins entendre mon introduction. $\mathrm{Ce}$ processus n'est-il pas à l'oeuvre dans l'élaboration de toute théorie scientifique ? Je n'ai pas osé aller aussi loin parce que les exemples que j' ai travaillés sont tous plus ou moins analogues à l'exemple 2 et semblent ressortir de la "modélisation". Mais je dois aussi avouer que je n'ai pas les idées très claires sur ce qu'est la "modélisation". Quelle différence y a-t-il entre un "modèle" et une "théorie scientifique"? Est-ce une différence de nature ou bien un "modèle" n'est-il qu'une micro-théorie? Je ne voulais pas m'avancer sur ce terrain mais la question se pose certainement.

Dans mes deux exemples je me suis livré à une reconstruction à posteriori d'un processus. Tout ne s'est certainement pas passé de manière aussi explicite que je le laisse entendre. N'est-ce pas moi qui vois, après coup, un processus rationnel là où il n'y a eu que le hasard des associations d'idées?

16. qui ont étés formulés par $\mathrm{G}$. Wallet qui a relu le texte initial et que je remercie ici pour ses contributions explicites et cachées à cette réflexion. 
S'il ne fait pas de doute que le processus de clarification-déplacement du sens des textesretour à l'interrogation est à l'oeuvre dans la pratique scientifique la question se pose de savoir s'il a bien toute l'importance que je lui accorde. Comme je l'ai dit, les deux exemples sur lesquels je me suis appuyé proviennent de ma seule expérience. S'il s'avérait que je sois le seul à pratiquer les élucidations successives cette méthode serait de bien peu d'intérêt. Comme le disait Sir Francis Crick $^{17}$ à l'auteur d'un exposé d'une théorie sur le cerveau :

This is not a theory of the brain, it is a theory of your brain!

\section{Bibliographie}

[1] R. BARBAUlt, « Ecologie des peuplements ». Masson, 1992.

[2] MacArthurR.H. And E.O. Wilson, «The theory of island biogeography » Evolution, $17: 373-387,2006$.

[3] G. HARDIN, « The competitive exclusion principle »Science, $131: 1292-1297,1960$.

[4] C. LOBRY, "Modèles mathématiques et modèles informatiques, sur la théorie du modèle » Annales des Ponts et Chaussée, 107-108:10, 2003.

[5] P. BRUNOVSKY AND C. LOBRY, «Contrôlabilité bang bang contrôlabilité différentiable et perturbation des systèmes non linéaires »Annali di matematica Pura ed Applicata(IV), CV :93$119,1975$.

[6] C. Lobry, A. Sciandra, And P. Nival, «Effets paradoxaux des fluctuations de l'environnement sur la croissance des populations et la compétition entre espèces »C. R. Acad. Sci. Paris, Sciences de la Vie, 317 :102-107, 1994.

[7] C. LobRY, «Modélisation d'effets paradoxaux en dynamique des populations » In Insitut de France, editor, Dynamiques des espèdes marines invasives, Actes de colloques, pages 4960. TEC DOC, London, Paris, New York, 1997.

[8] C. LOBRY AND G. WALLET, « Le passage de l'axe imaginaire n'a pas toujours lieu là où on croit l'observer » In M. Diener, editor, Actes du colloque "Mathématiques finitaires", Luminy 1985, pages 45-51. Université Paris VII, 1989.

[9] E. Benoit, B. CANDElPERGHeR, AND C. LOBRY, «Retard à la bifurcation en présence d'un bruit multiplicatif »Lecture notes in Control and Information Sciences, 122, 1988.

[10] C. LOBRY, «Sur le sens des textes mathématiques : Un exemple, la théorie des bifurcations dynamiques »Annales de l'Institut Fourier, 42 :327-351, 1992.

[11] M.A. SHISHKovA «Study of a system of differential equations with a small parameter at the highest derivatives »Dockl. Akad. Nauk SSSR, 209(3) :573-579, 1973.

[12] R. HABERMAN, « Slowly varying jump and transition phenomena associated with algebraic bifurcation problems »Siam. J. Applied. Math., 37(1) :69-106, 1976.

[13] L.A. RUBENFELD, "A model bifurcation problem exhibiting the effect of slow passage through critical » Siam J. Applied. Math., 37( $\left.\mathrm{n}_{i} 2\right): 573-579,1979$.

[14] P. MANDEL AND T. ERneUX, «Lazer lorenz equation with a time dependent parameter » Physical Review Letters, 53(19) :1818-1820, 2006.

[15] A.I. NeISHTADT, « Asymptotic study of stability loss of equilibrium under slow transition of two eigenvalues through critical point »Uspeki Mat. Nauk, 40(5):300-301, 1985.

17. Bien entendu c'est une histoire qu'on racconte, je ne sais si elle est vraie. 
[16] G. Wallet «Entrée sortie dans un tourbillon » Annales de l'Institut Fourier, 1986(364) :157-184, 1986.

[17] A. FRUChARD, « Retard à la bifurcation dans les systèmes dicrets » Séminaire Non Standard de Paris VI, 1989.

[18] ERIC BenOIT, EDITOR, Dynamical Bifurcations. Springer, Lecture Notes in Math., $n^{0} 1493$, 1991.

[19] G. WALLET, « De la bifurcation retardée à la surstabilité ou du différentiable réel à l'analytique complexe » Technical report, 1991. Thèse d'état, Université de Poitiers.

[20] J-L. CALlOT, « Champs lent-rapide complexes à une dimension lente»Ann. scient.Éc. norm. sup, 4 me série(26) :149-173, 1993.

[21] J. HARTHONG, «Eléments pour une théorie du continu »In Dynamical Bifurcation, volume 109-110, pages 235-244. Astérisque, 1983.

[22] I. LAKatos, Preuves et Réfutations. Herman, 1984.

[23] J-M. SALANSKIS, Le temps du sens. Editions HXY, 1997.

[24] J-M. SAlanskis, L'Herméneutique Formelle. Editions du CNRS, 1991.

[25] Salanskis J-M. F. RAstier ET R. Scheps, L’herméneutique : Les textes, la Science. PUF, 1997.

[26] Delattre P. ET M. Thellier, Editor Elaboration et Justification des Modèles. Maloine, Paris, 1979. 